\title{
Inductive coupler for contactless power transmission
}

\author{
Peter Sergeant and Alex Van den Bossche \\ Department of Electrical Energy, Systems and Automation, Ghent University \\ Sint-Pietersnieuwstraat 41, B-9000 Gent, Belgium \\ E-mail: Peter.Sergeant@UGent.be
}

\begin{abstract}
To provide power to a moving vehicle without using contacts, a transformer is presented, consisting of a fixed primary winding, inductively coupled to a moving secondary winding. The primary winding along the whole trajectory of motion is configured such that its magnetic stray field and its self inductance are limited. This winding transfers power to the moving coupler containing the yoke and the secondary winding. The secondary winding is a resonant circuit, with a rectifier and the motor of the vehicle as load. To describe the energy transmission system, a numerical model is developed that combines an electrical circuit with a 2D nonlinear finite element model. This model is used for an optimization of the design. For the optimized configuration, an experimental setup is built to validate the model by measurements. A sensitivity analysis is carried out concerning the primary current, the supply frequency, the position of the secondary winding relative to the primary winding and the number of vehicles coupled with the primary winding.
\end{abstract}

Keywords:

Transformers, Finite Element Analysis, Resonant Power Converters, Transmission Lines

\section{Introduction}

Several possible solutions are available to provide energy to moving or rotating systems such as electric vehicles, rotating electrical machines or robots. Examples are the commutator and brushes of a DC motor/generator and the catenary and pantograph of trains. A major disadvantage of these energy transfer systems concerns the contacts, which are subject to ageing and wear. Eventually, they limit the reliability of the device they supply energy to.

Transformers do not have these drawbacks, but they should be designed with care in order to reduce their volume, weight and losses. Such a design is carried out in [1] for a rotative transformer with iron core and for an ironless linear transformer.

Apart from the transfer of energy, the load often requires a suitable amplitude and waveform (DC, AC) of the voltage, so that a power electronics converter is needed to change the voltage 
waveforms. Both the contactless transfer of energy and the waveform conversion can be combined in a global design such as in the push-pull converter in [2]. The transformer designed in following paragraphs also combines both tasks, as it aims at a constant DC load voltage regardless of the input voltage. It is intended to supply - during service - an electrical motor or a battery recharger of automatic vehicles along a track.

The presented transformer is a "linear" transformer: its primary winding is a transmission line (TL) along the whole trajectory of motion - see Fig. 1. This transmission line has a fixed position, carries current with a frequency in the range $20-30 \mathrm{kHz}$ and transfers power to the moving "coupler". The latter contains the secondary winding and a magnetic yoke. The energy transmission system was developed for luggage transport with automatic vehicles in airports, as an alternative for the classical transport belts, which have a rather low energetic efficiency. With a rated power of $1 \mathrm{~kW}$, the system is designed for vehicles with a maximal weight (including luggage) of $100 \mathrm{~kg}$, a maximal speed of about $5 \mathrm{~m} / \mathrm{s}$ and the possibility to move on slopes up to $10 \%$ steepness.

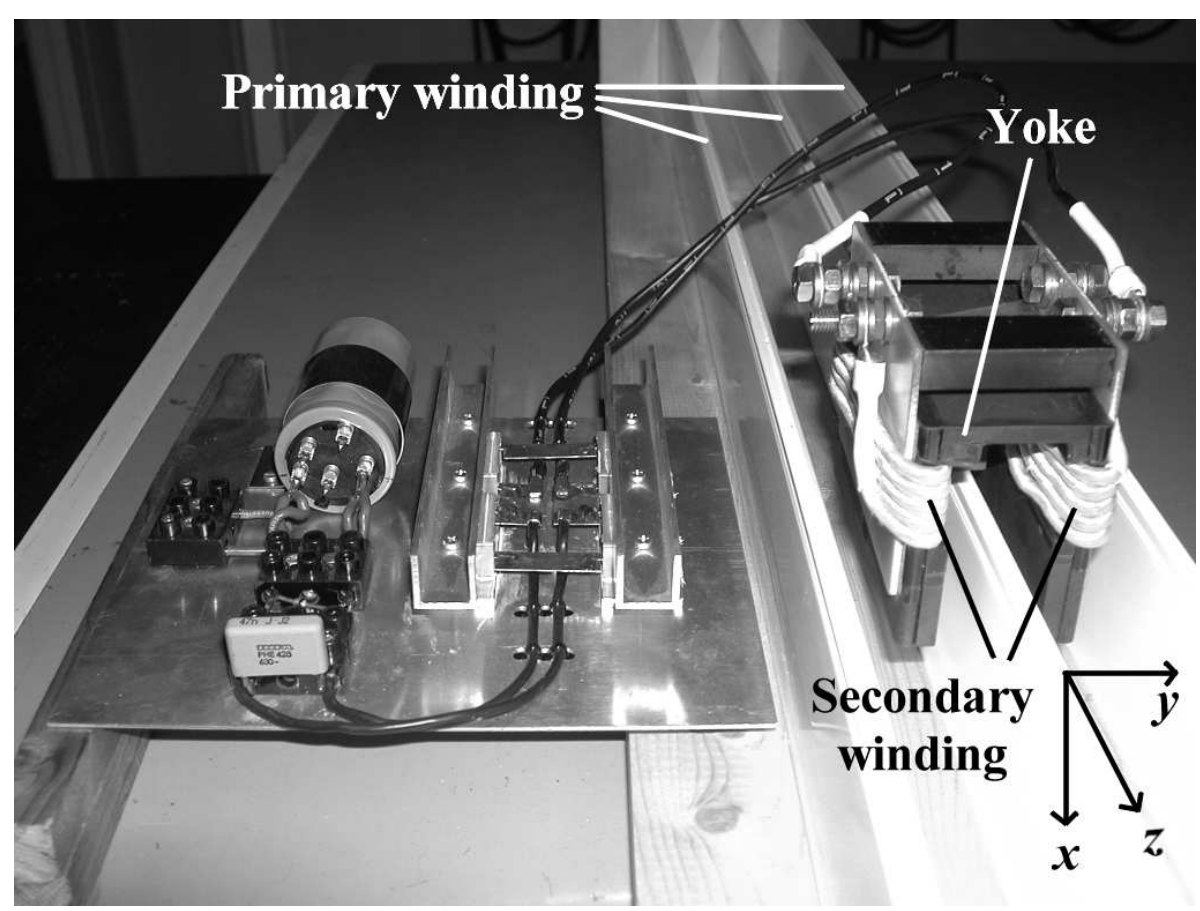

Figure 1: The primary and secondary winding of a linear transformer for contactless energy transmission, laying on its side (the $x$-axis is horizontal and the $y$-axis is vertical)

For this power transmission system, several design criteria are to be fulfilled.

- The energy transmission should be established whenever the coupler is "inside" the transmission line. There is no tight guidance that keeps the coupler at a fixed position relative to the transmission line. This means that the energy transmission should be robust against wrong positioning of the coupler in the plane perpendicular to the TL axis. Concerning 
the dimensions, the width of the transmission line should be about $100 \mathrm{~mm}$ and the coupler should be able to move in the plane perpendicular to the TL axis: $7.5 \mathrm{~mm}$ in both directions along the $y$-axis in Fig. 1 and even more along the $x$-axis.

- It should be possible to remove the coupler without any additional action such as the opening of a yoke. Hence, the coupler should not have a yoke that encloses the TL.

- The magnetic stray field of the transmission line should be limited in its neighbourhood.

- The coupler should have a high efficiency and good thermal behaviour for a rated power of $1 \mathrm{~kW}$ and a secondary voltage limited to $300 \mathrm{~V}$.

The paper firstly describes the model of the transformer. Secondly, the transformer is designed step by step by using the model, taking into account the above design criteria. Thirdly, the final experimental setup is outlined. Finally, several simulation and experimental results are given to illustrate the behaviour of the transformer. A sensitivity analysis is carried out to study the effect of variations in the number of vehicles, the supply frequency and the current in the TL.

\section{Model of the energy transmission system}

The energy transmission system consists of two mutually coupled coils with scheme shown in Fig. 2. Both the source connected to the TL (a half bridge) and the load (rectifier and motor) are nonlinear. For simplicity of the design routines however, we assume an ideal sinusoidal current source and a constant load impedance. Experiments show that, in spite of the simplifications, the energy transmission system complies with the design criteria. As we consider a sinusoidal source and a constant load, we solve the equations in the frequency domain:

$$
\begin{aligned}
& V_{1}=R_{1} I_{1}+\mathrm{j} \omega L_{1} I_{1}-\mathrm{j} \omega M_{12} I_{2} \\
& V_{2}=\mathrm{j} \omega M_{12} I_{1}-R_{2} I_{2}-\mathrm{j} \omega L_{2} I_{2}=Z_{\mathrm{L}} I_{2}
\end{aligned}
$$

The inductances $L_{1}, L_{2}$ and $M_{12}$ are on the one hand a function of the currents $I_{1}$ and $I_{2}$ that determine the saturation state of the ferrite core. On the other hand, they also depend on the geometry of the TL and the coupler and on the position $\left(x_{\mathrm{c}}, y_{\mathrm{c}}\right)$ of the coupler. The inductances are estimated using a 2D Finite Element Model (FEM) [3]. The model is static but nonlinear and has a mesh of 6200 elements. The calculated values of the inductances are only approximations as the 2D model considers the TL and the coupler as infinitely long objects. For the long TL, this approximation is acceptable, but for the rather short coupler, the neglecting of the fringing effects of the 3D geometry causes an underestimation of its self inductance. 


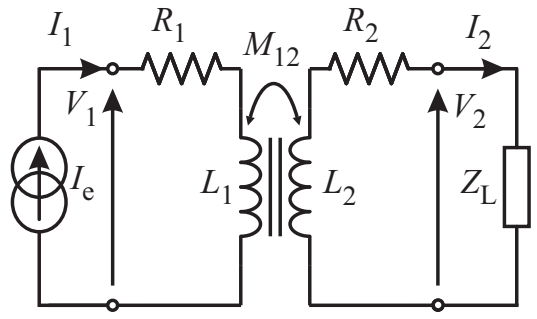

(a)

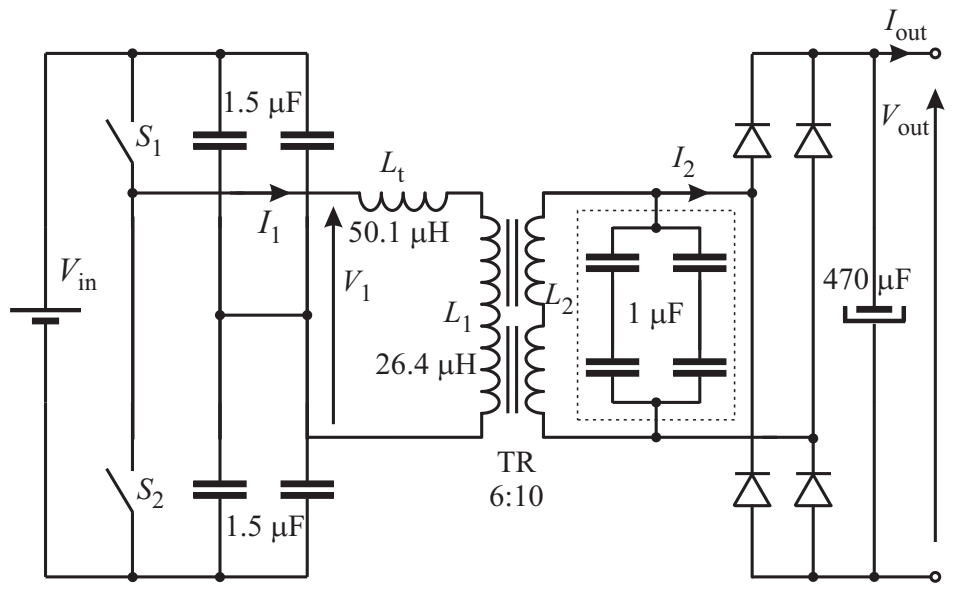

(b)

Figure 2: (a) General scheme of the two mutually coupled coils and (b) full scheme of the resonant circuit with the experimental values of the components ( $R_{1}$ and $R_{2}$ not shown)

The FEM needs a nonlinear magnetic characteristic of the ferrite yoke. Therefore, the $B-H$ or $H$ - $B$ relation is determined using the setup of [4] and fitted with an analytical expression

$$
\begin{aligned}
& B(H)=B_{\text {sat }} \tanh \left[\left(\frac{H}{H_{\text {knee }}}\right)^{g}\right] \\
& H(B)=H_{\text {knee }}\left[\operatorname{atanh}\left(\frac{B}{B_{\text {sat }}}\right)\right]^{1 / g}
\end{aligned}
$$

In the above equations, the $H$ and $B$ are peak values. For the ferrite $3 \mathrm{~F} 3$ [5], the parameters are $B_{\text {sat }}=0.32 \mathrm{~T}, H_{\text {knee }}=159 \mathrm{~A} / \mathrm{m}$ and $g=1.1$. A good correspondence is observed when comparing the experimental data with the calculated curve obtained from (3).

In the studied application - the supply for an electrical vehicle loading system - the current $I_{1}$ in the primary winding (the transmission line) is assumed to be imposed and the load impedance $Z_{\mathrm{L}}$ is known. As a result, the secondary voltage $V_{2}$ and current $I_{2}$ can be calculated from (2) if all components in the scheme are known:

$$
\begin{aligned}
I_{2} & =\frac{\mathrm{j} \omega M_{12}}{Z_{L}+R_{2}+\mathrm{j} \omega L_{2}} I_{1} \\
V_{2} & =Z_{\mathrm{L}} I_{2}
\end{aligned}
$$

The above equation (5) has to be solved iteratively as both $M_{12}$ and $L_{2}$ are function of the unknown $I_{2}$. The primary voltage is obtained from equation (1). For a given load impedance, a given geometry of the TL and the coupler and for a given position of the coupler, (5), (6) and (1) yield the output current and voltage, and the primary voltage as a function of the frequency. Afterwards, the power and the efficiency can be calculated as well. This information provided by the model will be used in section 3 to design the energy transmission system. 


\section{Design of the energy transmission system}

\subsection{A primary winding with low magnetic stray field}

For the primary winding, several possible configurations are compared for a given current rating in order to evaluate in the surrounding region the magnetic stray field which - according to the design criteria - should be minimal. The considered configurations are shown in Fig. 3 together with corresponding yoke shapes in the moving coupler. For the clarity of the figure, the yokes are drawn at too high distance from the primary winding and the secondary winding on the yoke is not shown. The three configurations are discussed.

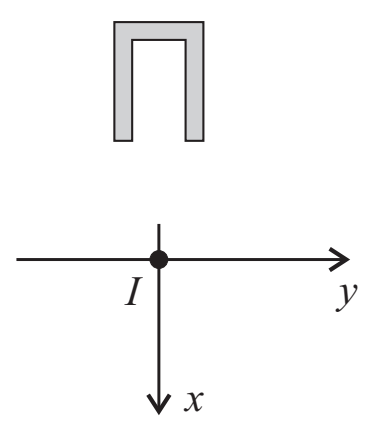

(a)

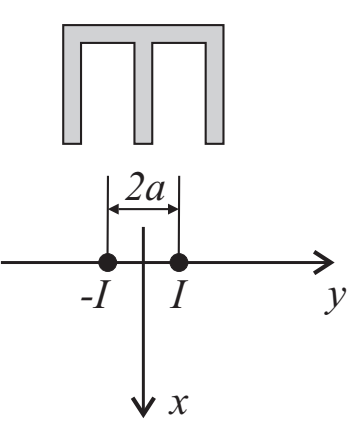

(b)
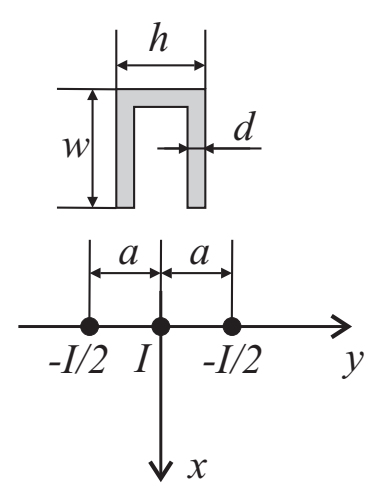

(c)

Figure 3: Possible configurations of the primary winding of the transformer and in gray corresponding geometries for the yoke of the moving coupler

(a) One conductor with current $I$ (Fig. 3a): the earth is used as return path. The coupler may consist of a U-core with one coil. Assuming that the return path is at rather large distance, the magnetic field

$$
H_{1}(x, y)=\frac{I}{2 \pi} \frac{-y \mathbf{1}_{x}+x \mathbf{1}_{y}}{x^{2}+y^{2}}
$$

can be simplified at a position along the $x$-axis as a function proportional to $1 / x$ :

$$
H_{1}(x, 0)=\frac{I}{2 \pi} \frac{1}{x} \mathbf{1}_{y}
$$

(b) Two conductors with opposite currents $I$ and $-I$ (Fig. 3b). Possible core shapes for the coupler are E-shaped cores or long I-cores. For two conductors at distance $2 a$ from each other, the magnetic field

$$
H_{2}(x, y)=\frac{I}{2 \pi} \frac{2 a\left(x^{2}+a^{2}-y^{2}\right) \mathbf{1}_{x}+4 a x y \mathbf{1}_{y}}{\left[x^{2}+(y-a)^{2}\right]\left[x^{2}+(y+a)^{2}\right]}
$$


is along the $x$-axis proportional to $1 / x^{2}$ for $x \gg a$ :

$$
H_{2}(x, 0)=\frac{I}{2 \pi} \frac{2 a}{x^{2}+a^{2}} \mathbf{1}_{x}
$$

(c) Three conductors in a plane with $I$ in the central one and $-I / 2$ in the outer ones

(Fig. 3c). A U-shaped core can be used for the coupler. The field

$$
H_{3}(x, y)=\frac{I}{2 \pi} \frac{-y a^{2}\left(3 x^{2}-y^{2}+a^{2}\right) \mathbf{1}_{x}+x a^{2}\left(x^{2}-3 y^{2}+a^{2}\right) \mathbf{1}_{y}}{\left[x^{2}+(y-a)^{2}\right]\left(x^{2}+y^{2}\right)\left[x^{2}+(y+a)^{2}\right]}
$$

decreases along the $x$-axis by $1 / x^{3}$ for $x \gg a$ :

$$
H_{3}(x, 0)=\frac{I}{2 \pi} \frac{a^{2}}{x\left(x^{2}+a^{2}\right)} \mathbf{1}_{y}
$$
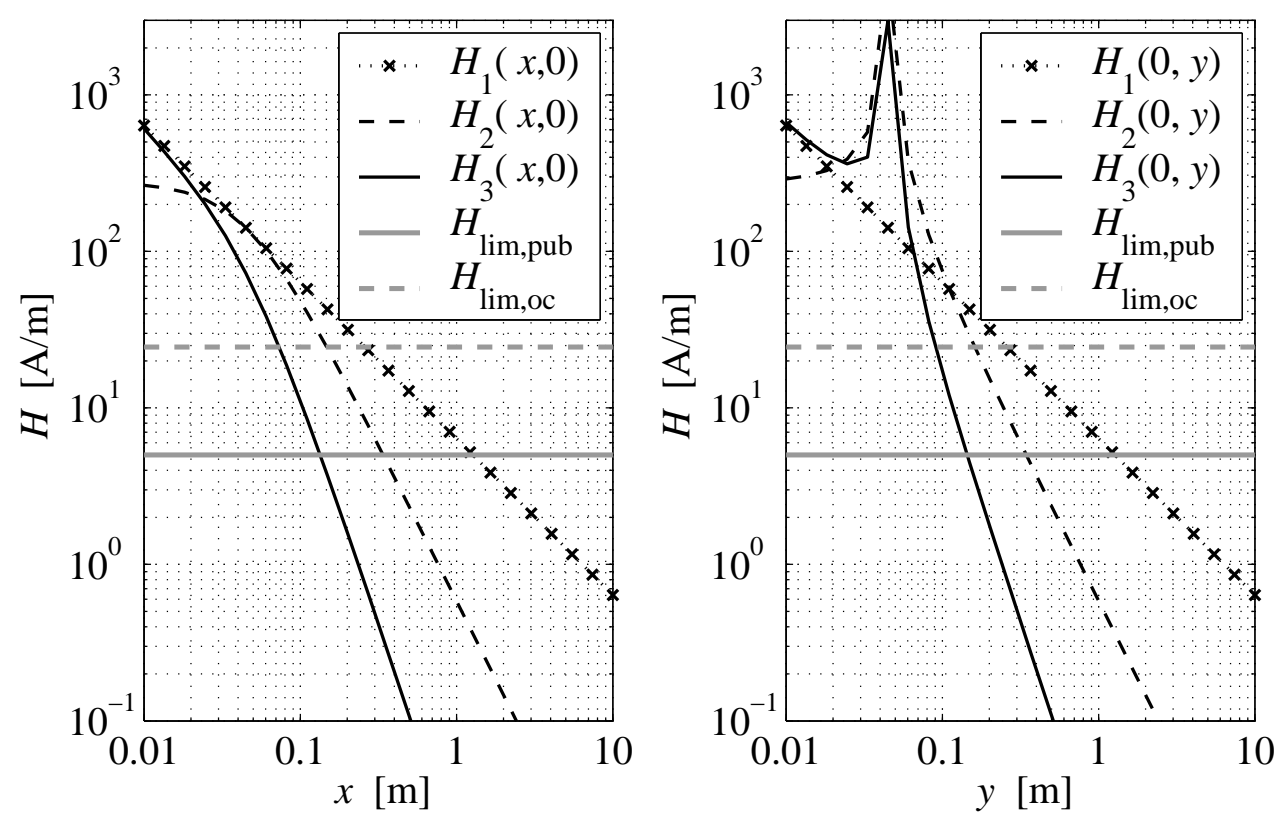

Figure 4: Magnetic stray field as a function of the distance (a) along the $x$-axis and (b) along the $y$-axis. The limits given by the European Community for occupational and general public exposure at the considered frequency are shown as well.

Fig. 4 shows the field of the three configurations as a function of the distance to the origin in Fig. 3. The fields are calculated in absence of possible magnetic or conductive construction materials in the neighbourhood. The curves are obtained using $I=40 \mathrm{~A}$. Because of the maximal total width of $100 \mathrm{~mm}$ specified in the design criteria and because of construction aspects, the parameter $a$ is chosen equal to $36 \mathrm{~mm}$. These values are the ones used in the experimental setup.

Due to the low stray field, configuration c is selected for the primary winding. To not exceed the reference values from the European Community, the safety distance should be at least $9 \mathrm{~cm}$ for 
occupational exposure $(24.4 \mathrm{~A} / \mathrm{m}$ at $25 \mathrm{kHz})$ [6] or $14 \mathrm{~cm}$ for general public exposure $(5 \mathrm{~A} / \mathrm{m}$ at $25 \mathrm{kHz}$ ) [7]. For the situation with one conductor, this distance should be more than $1 \mathrm{~m}$.

The final lay-out of the primary winding is shown in Fig. 5a and in Fig. 1. The middle bar with total current $I$ consists of two pairs of three conductors with current $I / 6$ each. The bar has a total width of $10 \mathrm{~mm}$. Other construction details can be found in section 4.1. The U-shaped yoke and secondary winding are optimized in the following section.

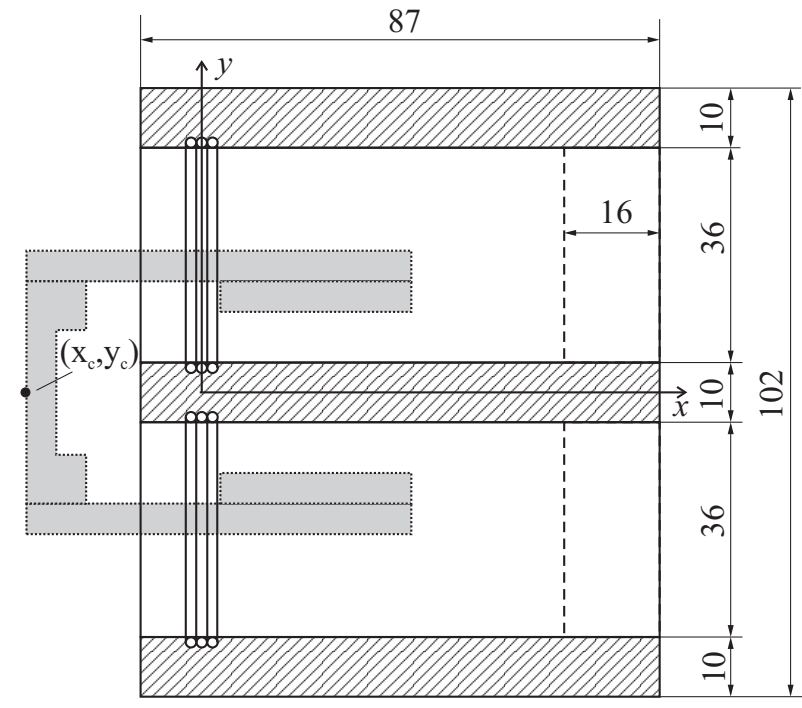

(a)

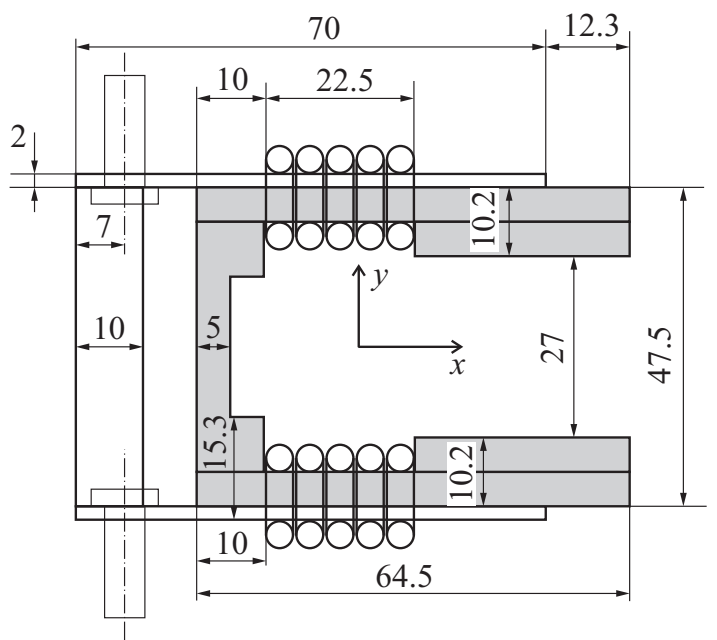

(b)

Figure 5: (a) Geometry of the cross section of the primary winding and (b) of the coupler with ferrite core and secondary winding; dimensions in millimeters. The axial length $l_{\mathrm{c}}$ is $100 \mathrm{~mm}$.

\subsection{A secondary winding and a U-shaped core, robust against positioning errors}

The width $w$ and height $h$ of the U-core (see Fig. 3c) should be optimized. These parameters are a compromise between on the one hand sufficient tolerance for a bad positioning of the coupler relative to the middle bar (of $10 \mathrm{~mm}$ width) containing the middle primary conductors and on the other hand a low magnetic reluctance. For three heights $h$, Fig. 6 shows the influence of the width $w$ by displaying the mutual inductance per meter axial length $m_{12}$ between primary and secondary winding obtained by FEM, for a primary current of $6.67 \mathrm{~A}$ (40 A for the $2 \times 3$ turns together). To calculate $m_{12}$, we assumed a secondary winding with 10 turns and a primary winding of 6 turns. It is evident that $m_{12}$ should be maximal. In Fig. $3 \mathrm{c}, d$ is $10 \mathrm{~mm}$.

The height $h$ should be much higher than $2 d$ to enable sufficient motion along the $y$-axis. Because of design criteria and the TL geometry, $h$ should be at least $45 \mathrm{~mm}$. Furthermore, as $m_{12}$ decreases with increasing $h$, the height $h$ should be as low as possible. As a compromise and taking into account the size of commercially available sizes of ferrites, the choice made for the 


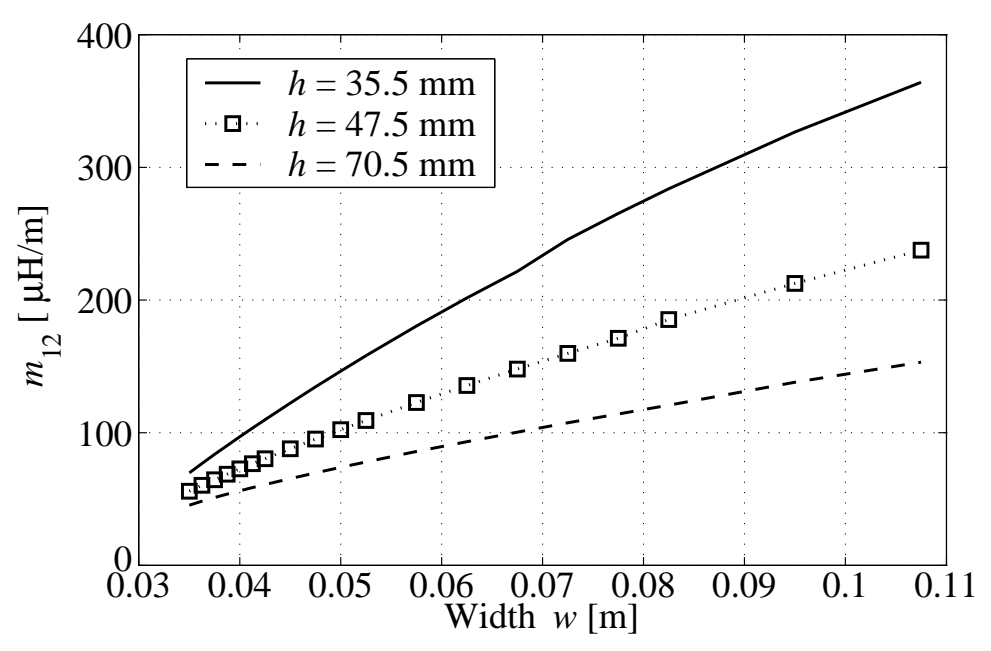

Figure 6: Mutual inductance $m_{12}$ per $m$ axial length as a function of the width $w$ and height $h$ of the yoke

height is $47.5 \mathrm{~mm}$. A similar approach for the width $w$ leads to the eventual choice of $64.5 \mathrm{~mm}$. The final geometry of the yoke is shown in Fig. $5 b$.

The robustness against positioning errors of the yoke is shown in Fig. 7. It is seen that $m_{12}$ is sufficient if the legs of the core are between the TL conductors, i.e. if $-x_{\mathrm{c}} \leq w=64.5 \mathrm{~mm}$.

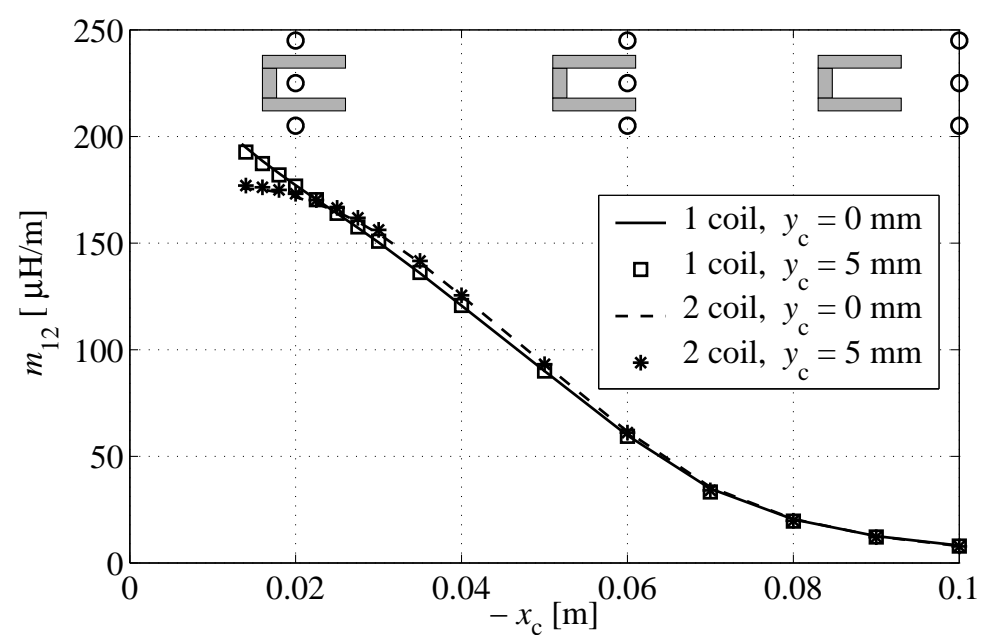

Figure 7: Mutual inductance $m_{12}$ per $m$ axial length as a function of the horizontal and vertical position of the yoke containing one secondary winding on the vertical left part of the yoke in Fig. $5 b$ or two secondary windings in series on the two horizontal legs as shown in Fig. $5 b$

The geometry and the position on the yoke of the secondary winding have also been studied in order to increase this robustness against bad positioning of the coupler. The possibilities are either one coil on the left vertical leg in Fig. 5b, or two coils on both horizontal legs as shown in Fig. 5b. Concerning the horizontal position $x_{\mathrm{c}}$ of the coupler, it can be seen from Fig. 7 that with only one coil the mutual inductance continues to increase if $x_{\mathrm{c}} \rightarrow 0$, i.e. if the TL is very close to the vertical left leg of the yoke. In order to have less sensitivity of $m_{12}$ to $x_{\mathrm{c}}$ and also 
because of thermal reasons, two coils were chosen. A variation of the vertical position $y_{\mathrm{c}}$ of the coupler has almost no influence on $m_{12}$, neither in case of one coil nor in case of two coils.

The axial length and the number of turns were chosen in order to obtain a secondary voltage of maximally $300 \mathrm{~V}$ as mentioned in the design criteria. The secondary voltage is $V_{2}=\mathrm{j} \omega n_{\mathrm{c}} \phi$ where the maximal flux in saturation is $\phi=B_{\mathrm{sat}} l_{\mathrm{c}} d_{\mathrm{c}}$. With ferrite of thickness $d_{\mathrm{c}}=5 \mathrm{~mm}$, the number of turns $n_{\mathrm{c}}=2 \times 5$ and $B_{\mathrm{sat}} 0.32 \mathrm{~T}$, we find that $l_{\mathrm{c}}$ should be $106 \mathrm{~mm}$ at a frequency of e.g. $28 \mathrm{kHz}$. Again taking into account the commercially available sizes, the axial length in the experimental setup is chosen equal to $100 \mathrm{~mm}$. The calculated self inductance is $32 \mu \mathrm{H}$.

\subsection{Higher power output due to resonant circuit}

The choice for a resonant circuit instead of a classical current transformer with rectifier load, makes it possible to increase the active power transferred to the load for a frequency close to the resonance frequency. The scheme is similar to the one of the superconducting transformer for DC/DC power conversion in [8]: we consider a capacitor of $1 \mu \mathrm{F}$ that together with the transformer's self inductance (leakage inductance plus magnetizing inductance) of $32 \mu \mathrm{H}$ causes a resonance at $28 \mathrm{kHz}$. As the inductance is calculated by a 2D-approximation, the experimental resonance frequency may be different. The resonance frequency is the result of a compromise: on the one hand, this frequency should be maximal in order to maximize the output voltage and power; on the other hand, this frequency should be low enough in order to limit the dissipation and the temperature of the saturated ferrite yoke and to guarantee a small safety distance regarding the stray field of the primary winding. Indeed, the reference levels of the European Community decrease with increasing frequency.

The impedance of the coupler with capacitor is near the resonance frequency much higher than the impedance of the coupler without the capacitor, resulting in a higher power transfer. To avoid overvoltages, the saturation of the ferrite core is exploited: due to saturation, the flux through the core and through the windings of the coupler is limited in a natural way. There is no need for additional protection against overvoltage.

If the small series resistances $R_{1}$ and $R_{2}$ are neglected, the equations (1) and (2) are:

$$
\begin{aligned}
V_{1} & =\mathrm{j} \omega L_{1} I_{1}-\mathrm{j} \omega M_{12} I_{2} \\
V_{2} & =\mathrm{j} \omega M_{12} I_{1}-\mathrm{j} \omega L_{2} I_{2}=\frac{Z_{L}}{\mathrm{j} \omega C Z_{L}+1} I_{2}
\end{aligned}
$$

As the current $I_{1}$ is imposed and the load impedance $Z_{L}$ is assumed to be known, we obtain:

$$
I_{2}=\frac{\mathrm{j} \omega M_{12}\left(\mathrm{j} \omega Z_{L} C+1\right)}{Z_{L}+\mathrm{j} \omega L_{2}-\omega^{2} Z_{L} L C}
$$


By iteratively solving the non-linear equation (15) in combination with $V_{2}=Z_{L} I_{2}$, the Fig. 8 is generated, showing the secondary voltage for several primary currents, no-load and neglected resistances $R_{1}$ and $R_{2}$. The resonance frequency at $28 \mathrm{kHz}$ is observed. It is seen that even for low $I_{1}$, the maximal output voltage can be achieved if the supply frequency is close to this resonance frequency ${ }^{1}$. For higher $I_{1}$, the maximal voltage is achieved also if the frequency is somewhat different from the resonance frequency. Furthermore, the figure shows that - due to the saturating ferrite - the output voltage never exceeds $280 \mathrm{~V}$ although all resistances that might damp the resonance are neglected.

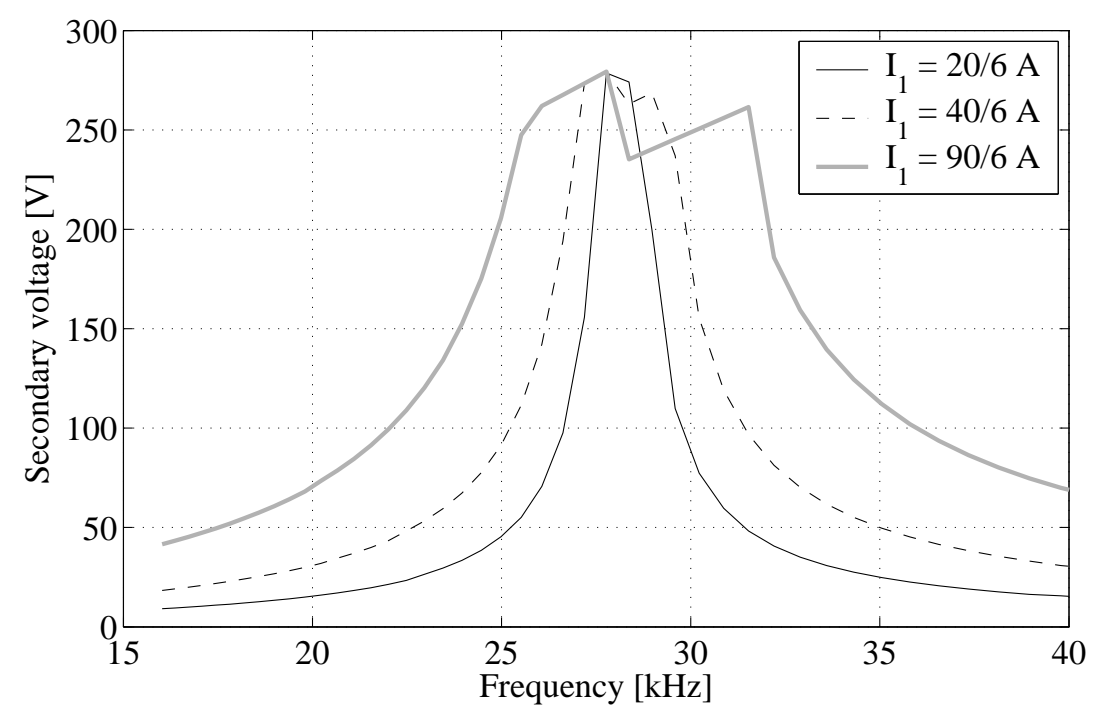

Figure 8: Secondary voltage $V_{2}$ as a function of the frequency for several primary currents $I_{1}$

\section{Experimental setup}

The linear transformer was built according to the design guidelines discussed in the previous section. The paragraph 4.1 lists a detailed description of the experimental setup shown in Fig. 1. Paragraph 4.2 explains the complete electrical scheme of the transformer in Fig. $2 \mathrm{~b}$ and compares the measured and calculated values of the impedances.

\subsection{Construction details}

The supply to the primary winding is a half bridge converter $[9,10]$ with $2 \times 2 \times 1.5 \mu \mathrm{F}$ coupling capacitors. By adding a series inductor to the primary winding, the supply behaves more or less like a current source.

\footnotetext{
${ }^{1}$ In practice, the resonance frequency is tuned and the supply frequency is fixed
} 
For the primary winding (Fig. 5a), the current of typical $6.7 \mathrm{~A}$ in combination with the frequency of $20-30 \mathrm{kHz}$ (penetration depth in copper is only $0.45 \mathrm{~mm}$ ) requires the use of Litz wire. The chosen Litz wire with a total section of $1.06 \mathrm{~mm}^{2}$ consists of 60 insulated copper wires (strands) with $0.15 \mathrm{~mm}$ diameter. Its resistance is $18.9 \mathrm{~m} \Omega / \mathrm{m}$ and results in $0.84 \mathrm{~W} / \mathrm{m}$ losses for 6.7 A current. In the setup, the primary winding is $1 \mathrm{~m} \mathrm{long}$, has $2 \times 3$ turns in Litz wire and a self inductance of $26.3 \mu \mathrm{H}$. The extra self inductance $L_{\mathrm{t}}$ (Fig. 2b) was added in order to simulate a primary winding longer than $1 \mathrm{~m}$. With $L_{\mathrm{t}}=50 \mu \mathrm{H}$, about $2 \mathrm{~m}$ extra length of the primary winding is simulated.

The magnetic core of the coupler (Fig. 5b) was made from 6 planar I-profiles and one cut E profile in the material $3 \mathrm{~F} 3$, all having $5 \mathrm{~mm}$ thickness. Taking into account an axial length $l_{\mathrm{c}}$ of $100 \mathrm{~mm}$ and a saturation of the ferrite of $0.32 \mathrm{~T}$, the maximal flux $\phi_{\max }$ through the core is $160 \mu \mathrm{Wb}$. At $25 \mathrm{kHz}$ frequency for example, this flux in the coupler core causes a voltage drop of $\mathrm{j} \omega \phi_{\max }=25.1 \mathrm{~V}$ peak per turn in the primary winding. Hence, the number of couplers that can take energy from the primary winding is limited by the voltage of the supply converter of the primary winding.

The secondary winding consists of ten turns in Litz wire with 760 insulated copper strands of $0.1 \mathrm{~mm}$ diameter (copper cross section is $5.97 \mathrm{~mm}^{2}$ and resistance is $4.0 \mathrm{~m} \Omega / \mathrm{m}$ ) resulting in a self inductance of $40 \mu \mathrm{H}$. The wire is chosen thicker than for the primary winding because the current in the resonant circuit approximates $34.5 \mathrm{~A}$. At $25 \mathrm{kHz}$, the winding with ten turns produces a maximal peak voltage of $274 \mathrm{~V}$ at $100^{\circ} \mathrm{C}$. For lower temperatures, the voltage may be $10 \%$ higher.

The resonant circuit is established by adding a capacitance of $1 \mu \mathrm{F}$ in parallel to the secondary winding. The resonance frequency $1 /\left(2 \pi \sqrt{L_{2} C}\right)$ was measured to be $24.7 \mathrm{kHz}$. As expected, this is lower than the calculated resonance frequency of $28 \mathrm{kHz}$, because the experimental self inductance $L_{2}$ is larger than the one calculated by the 2D FEM. The capacitance is realized by 4 capacitors of $1 \mu \mathrm{F}$ mounted on a heatsink to avoid overheating of the capacitors with a loss factor of $0.3 \%$.

The load is a diode rectifier ( $4 \times \mathrm{BYW} 95)$ with a capacitor of $470 \mu \mathrm{F}$ at the DC side, connected with a load resistor. The commutation of the diodes is zero voltage switch, so that the losses are mainly caused by the currents during conduction and not by the switching of the diodes.

\subsection{Complete electrical scheme}

A comparison is made between the calculated and measured impedances in the scheme of Fig. 2. The inductances are a function of the primary and secondary currents and the position $\left(x_{c}, y_{c}\right)$ 
of the coupler.

- The resistances $R_{1}$ and $R_{2}$ can be calculated quite accurately using the resistances of the Litz wire given in section 4.1. As the primary winding has twelve conductors (see Fig. 5a) of $18.9 \mathrm{~m} \Omega / \mathrm{m}$ each, we obtain $R_{1}=0.226 \Omega$ for the $1 \mathrm{~m}$ long line. The secondary winding has twenty conductors (see Fig. 5b) in Litz wire of $4 \mathrm{~m} \Omega / \mathrm{m}$ and an axial length of $100 \mathrm{~mm}$, resulting in $R_{2}=0.080 \Omega$.

- As the self inductance $L_{1}$ depends on the primary current $I_{1}$ and the position of the coupler, we compare measurements and simulations for a chosen weak current $(0.01 \mathrm{~A})$ and a coupler at a position $(-0.15,0)$, far from the TL. The measured self inductance is $26.4 \mu \mathrm{H}$ for the one meter long transmission line. The calculated self inductance is $27.3 \mu \mathrm{H}$, which is only $3 \%$ higher.

- The self inductance $L_{2}$ was measured to be $40 \mu \mathrm{H}$. The calculated self inductance was $319 \mu \mathrm{H}$ per meter. Taking into account the axial length $l_{c}$ of $0.1 \mathrm{~m}$, the calculated $L_{2}$ is $31.9 \mu \mathrm{H}$, which is $20 \%$ underestimated by the $2 \mathrm{D}$ FEM because the fringing effects in the $z$-direction are not modelled.

- The mutual inductance $M_{12}$ is calculated per meter axial length $m_{12}=M_{12} / l_{\mathrm{c}}$ for several situations in Fig. 7. As again fringing in the $z$-direction is neglected, the experimental values are higher than the calculated ones.

\section{Experiments}

\subsection{Without coupler}

Firstly, we consider the transmission line without coupler and with a square voltage waveform, not a sinusoidal one like in the simulations. The oscilloscope depicts - because of the inductive $\mathrm{TL}$ - a triangular current waveform. Tables 1 to 3 show the DC-value of the input voltage $V_{\text {in }}$ and current $I_{\text {in }}$, the DC (rectified) output voltage $V_{\text {out }}$ and current $I_{\text {out }}$ measured at the load, the input power $P_{\text {in }}$ in the TL and the output power $P_{\text {out }}$ to the load, and the efficiency $\eta$. Without coupler (Table 1), the input power is dissipated in the TL.

\subsection{With coupler at no-load}

At no-load, but with the coupler close to the TL, the same quantities as in section 5.1 are given in Table 2. As there is no load, evidently, the output current, power and efficiency are zero. 
Table 1: For the transmission line without coupler: input voltage and current, and input power

\begin{tabular}{ccccccc}
\hline $\begin{array}{c}V_{\text {in }} \\
{[\mathrm{V} \mathrm{DC}]}\end{array}$ & $\begin{array}{c}I_{\text {in }} \\
{[\mathrm{A} \mathrm{DC}]}\end{array}$ & $\begin{array}{c}V_{\text {out }} \\
{[\mathrm{V} \mathrm{DC}]}\end{array}$ & $\begin{array}{c}I_{\text {out }} \\
{[\mathrm{A} \mathrm{DC}]}\end{array}$ & $\begin{array}{c}P_{\text {in }} \\
{[\mathrm{W}]}\end{array}$ & $\begin{array}{c}P_{\text {out }} \\
{[\mathrm{W}]}\end{array}$ & $\begin{array}{c}\eta \\
{[\%]}\end{array}$ \\
\hline 100 & 0.113 & - & - & 11.3 & - & - \\
200 & 0.198 & - & - & 28.0 & - & - \\
250 & 0.245 & - & - & 73.5 & - & - \\
\hline
\end{tabular}

Table 2: For the transmission line without coupler: input voltage and current, output voltage and current, input and output power, and efficiency $\eta$

\begin{tabular}{ccccccc}
\hline $\begin{array}{c}V_{\text {in }} \\
\text { [V DC] }\end{array}$ & $\begin{array}{c}I_{\text {in }} \\
\text { [A DC] }\end{array}$ & $\begin{array}{c}V_{\text {out }} \\
\text { [V DC] }\end{array}$ & $\begin{array}{c}I_{\text {out }} \\
\text { [A DC] }\end{array}$ & $\begin{array}{c}P_{\text {in }} \\
{[\mathrm{W}]}\end{array}$ & $\begin{array}{c}P_{\text {out }} \\
{[\mathrm{W}]}\end{array}$ & $\begin{array}{c}\eta \\
{[\%]}\end{array}$ \\
\hline 100 & 0.090 & 124 & 0 & 9.0 & 0 & 0 \\
200 & 0.183 & 249 & 0 & 24.9 & 0 & 0 \\
250 & 0.218 & 304 & 0 & 54.5 & 0 & 0 \\
300 & 0.220 & 336 & 0 & 66.0 & 0 & 0 \\
\hline
\end{tabular}

\subsection{With coupler and load}

By comparing the data in Table 3 for input voltages of $250 \mathrm{~V}$ and $300 \mathrm{~V}$, the effect of the saturation of the ferrite on $V_{\text {out }}$ can be observed. The efficiency is about $81-86 \%$. The section 6 mentions some improvements in order to obtain an efficiency of $90 \%$ or more.

Table 3: For the transmission line with coupler and load for an input voltage of either $250 \mathrm{~V}$ or $300 \mathrm{~V}$ : input voltage and current, output voltage and current, input and output power, and efficiency $\eta$

\begin{tabular}{ccccccc}
\hline $\begin{array}{c}V_{\text {in }} \\
\text { [V DC] }\end{array}$ & $\begin{array}{c}I_{\text {in }} \\
\text { [A DC] }\end{array}$ & $\begin{array}{c}V_{\text {out }} \\
{[\text { [V DC] }}\end{array}$ & $\begin{array}{c}I_{\text {out }} \\
{[\text { [A DC] }]}\end{array}$ & $\begin{array}{c}P_{\text {in }} \\
{[\mathrm{W}]}\end{array}$ & $\begin{array}{c}P_{\text {out }} \\
{[\mathrm{W}]}\end{array}$ & $\begin{array}{c}\eta \\
{[\%]}\end{array}$ \\
\hline 250.0 & 1.41 & 290.8 & 1.0 & 352.5 & 290.8 & 82.4 \\
250.5 & 2.45 & 265.3 & 2.0 & 613.7 & 530.6 & 86.4 \\
250.1 & 3.02 & 216.0 & 3.0 & 755.3 & 648.0 & 85.5 \\
\hline 300.3 & 1.31 & 321.4 & 1.0 & 393.4 & 321.4 & 81.7 \\
300.6 & 2.35 & 303.5 & 2.0 & 706.4 & 607.0 & 86.5 \\
301.8 & 3.25 & 281.6 & 3.0 & 980.8 & 844.8 & 86.1 \\
\hline
\end{tabular}




\section{Robustness analysis and possible improvements}

\subsection{Number of couplers}

In the studied application, it is possible that several vehicles need a power supply simultaneously and on the same TL. As mentioned earlier, each coupler causes a voltage drop per turn in the primary winding that equals $\mathrm{j} \omega \phi_{\max } \approx 25 \mathrm{~V}$ near the resonance frequency of $24.7 \mathrm{kHz}$ (measured). In the experimental setup, the primary winding consists of $2 \times 3$ turns (Fig. 5a) so that the voltage drop per coupler equals $6 \times 25 \mathrm{~V}=150 \mathrm{~V}$ peak. The experimental setup has been designed to test only one coupler. Therefore, a DC supply voltage $V_{\text {in }}$ of $250 \mathrm{~V}$ is chosen, which results in $159 \mathrm{~V}$ amplitude of the fundamental component of the voltage waveform produced by the half bridge converter.

If energy has to be transferred to more couplers simultaneously, then two easy solutions are either to provide a higher supply voltage $V_{\text {in }}$, or to replace the $2 \times 3$-turn primary winding by a single-turn coil using Litz wire with more strands or a copper tube. In both cases, a more powerful converter is needed.

However, if some of the couplers are in no-load, the maximal number can be increased without changing $V_{\text {in }}$. Indeed, the couplers in no-load are almost pure inductances. The voltage drop they cause can be reduced by adding a series capacitor to the transmission line.

\subsection{Variation of primary current}

Although by using the resonance, the maximal secondary voltage can be achieved in a wide range of $I_{1}$, there are some disadvantages about a very low or very high primary current.

With a low primary current, the tolerance concerning the frequency is very small. Moreover, a large yoke must be used that causes a high voltage drop in the TL. Indeed, the power $P_{2}$ is always lower than the product of $I_{1}$ and the voltage drop. In other words, if $I_{1}$ is limited, then also the available power is limited.

A very high $I_{1}$ causes high losses and a high voltage drop per meter in the TL due to its series resistance, as well as a high magnetic stray field in the neighbourhood.

An optimal choice of the current can be achieved by implementing feedback of the couplers about their secondary voltage. If the worst-case coupler has enough voltage, $I_{1}$ can be reduced. 


\subsection{Variation of frequency}

The operational frequency has been chosen as a compromise between high power transmission (which requires high frequency) and acceptable ferrite losses at saturation (which limits the maximal frequency). In case of resonance, Fig. 8 shows that the obtained voltage is almost independent of the frequency in a small frequency range. The core is always saturated due to the high secondary current. The voltage is $274 \mathrm{~V}$ measured (at $100^{\circ} \mathrm{C}$ ), which corresponds to Fig. 8 for 40 A primary current. A large variation of the supply frequency may cause a high decrease of voltage in the secondary winding. It seems interesting to choose a high primary current, as this broadens the frequency range in which saturation occurs. However, as already mentioned, a higher primary current causes a higher voltage drop and loss in the primary winding.

\subsection{Efficiency}

The efficiency is about $85 \%$ under normal load conditions - see Table 3. Improving the efficiency requires the reduction of the three main loss contributions: Joule losses due to the resistance of the primary and secondary winding, electromagnetic losses in the ferrite core and losses in the capacitors in the resonant circuit. The first loss term can be reduced by choosing for the primary and secondary winding Litz wire with more strands. Also the last loss contribution may be reduced by replacing the capacitors with a loss factor of $0.3 \%$ by other ones with $0.03 \%$ loss factor. This replacement also eliminates the need for cooling of the capacitors.

\section{Conclusion}

The energy transmission system supplies inductive energy to a moving coupler that doesn't enclose the transmission line. Simulations of the system firstly discuss design aspects using a simplified model. This model describes the system for sinusoidal waveforms instead of square waves and 2D instead of 3D. Secondly, experiments show that the energy transmission system meets the design criteria. Because of the low stray field, the required safety distance is low. It is shown that the system is not very sensitive to variations of the primary current and voltage, and to bad positioning of the coupler. The saturation of the coupler effectively limits the output voltage. Some indications are given to improve the efficiency which is about $85 \%$ under normal load conditions. The efficiency is high enough to consider an application for recharging automatic guided vehicles (on tracks) during service. 


\section{Acknowledgement}

The first author is a postdoctoral researcher of the "Fonds voor Wetenschappelijk Onderzoek Vlaanderen" (FWO).

\section{References}

[1] Jufer, M., Macabrey, N., and Perrottet, M.: "Modeling and test of contactless inductive energy transmission", Mathematics and Computers in Simulation, 1998, 46, pp. 197-211

[2] Chen, R. T., and Chen, Y.Y.: "Synthesis and design of integrated-magnetic-circuit transformer for VRM application", IEE Proc. Power Appl., 2006, 153, (3), pp. 369-378

[3] Silvester, P. P., and Ferrari, R. L.: "Finite elements for electrical engineers" (Cambridge University Press, Cambridge, 1990)

[4] Valchev, V., Van den Bossche, A., and Van de Sype D.: "Ferrite losses of cores with square wave voltage and DC bias", Proc. IECON 2005 - The 31st Annual Conference of the IEEE Industrial Electronics Society, Raleigh, North Carolina, 6-10 Nov. 2005

[5] Ferroxcube Datasheets: "EP cores and accessoiries", 2004

[6] European Council: "Directive 2004/40/EC of the European parliament and of the council of 29 April 2004 on the minimum health and safety requirements regarding the exposure of workers to the risks arising from physical agents (electromagnetic fields)", Official Journal of the European Union, 2004, L 184, pp. 1-9

[7] European Council: "Council recommendation of 12 July 1999 on the limitation of exposure of the general public to electromagnetic fields (0 Hz to $300 \mathrm{GHz}$ )", Official Journal of the European Union, 1999, L 199, pp. 59-70

[8] Cheng, K. W. E., Chan, H. L., and Sutanto, D.: "Development of a superconducting transformer using self-resonant techniques for DC/DC power", IEE Proc. Power Appl., 2004, 151, (3), pp. 296-302

[9] Mohan, N., Undeland, T. M., and Robbins, W. P.: "Power electronics: converters, applications and design" (John Wiley \& Sons, New York, 1995)

[10] Van den Bossche, A., Filchev, T., Valchev, V., and Yudov, D.: “Test Platform for Resonant Converters", Proc. EPE Conference, Toulouse, France, 3 - 4 Sep. 2003, cd-rom 